\title{
Reversal of Dibromothymoquinone Inhibition of Photosynthetic Electron Transport by Bovine Serum Albumin ${ }^{1}$
}

\author{
H. H. ROBINSON, J. A. GUIKEMA, ${ }^{2}$ AND C. F. YOCUM \\ Division of Biological Sciences, The University of Michigan, Ann Arbor, Michigan 48109
}

Received February 26, 1980

\begin{abstract}
Addition of bovine serum albumin to cholorplasts inhibited by prior addition of $1 \mu \mathrm{M}$ dibromothymoquinone results in a time- and light-dependent restoration of electron transport activity. The kinetics of this reversal reaction are complex, and indicate that it is controlled by the degree to which the thylakoid membranes are energized. The presence of ADP and inorganic phosphate, or of uncouplers, serves to retard the rate of reversal, whereas an acceleration of reversal is observed if the thylakoid membranes have been intentionally unstacked by exposure to low-salt medium. The reversal reaction reported here is unique to bovine serum albumin, and does not require the function of the free sulfhydryl group on the protein. It is concluded that the site of DBMIB inhibition associated with chloroplast membranes is situated in a position whose access to contact by bovine serum albumin is regulated by the structural changes induced by illumination and energization.
\end{abstract}

At low concentrations $(\leq 1 \mu \mathrm{M})$, dibromothymoquinone (DBMIB) ${ }^{3}$ inhibits photosynthetic electron transport in isolated chloroplasts at a site located between photosystem II and photosystem I (1); the inhibition is presumed to arise from interference with the oxidation of plastoquinone (2). Higher concentrations of DBMIB $(>3 \mu \mathrm{M})$ exert an inhibitory influence on chloroplast electron transport at a second site close to the reducing side of photosystem II (3-6). Guikema and Yocum (6) characterized the differences between the high-affinity $(\leq 1$ $\mu \mathrm{M})$ and low-affinity ( $>3 \mu \mathrm{M})$ sites of DBMIB inhibition by showing that the thiolmediated reversal of inhibition at these sites by thiols differed both with regard to the thiol/DBMIB ratio necessary to affect

${ }^{1}$ This research was supported by a grant from the National Science Foundation (PCM78-7909).

${ }^{2}$ Present address: Division of Biological Sciences, University of Missouri, Columbia, Mo. 65211.

${ }^{3}$ Abbreviations used: DBMIB, 2,5-dibromo-3methyl-6-isopropyl-p-benzoquinone; BSA, bovine serum albumin; BSA-CAM, carboxymethylated bovine serum albumin; Val, valinomycin; Gram, gramicidin; PS, photosytem; chl, chlorophyll; DTT, dithiothreitol; $\mathrm{MV}$, methylviologen; $\mathrm{I}_{50}$, concentration of inhibitor required for $50 \%$ inhibition of electron transport activity. reversal, and with regard to the time of dark exposure to thiol required for reversal of inhibition. It was also shown that BSA was capable of partially reversing the inhibition of electron transport by DBMIB at the low-affinity site; no such reversal of inhibition at the high-affinity inhibition site was observed in these experiments.

The ability of BSA to bind DBMIB in solution, and thus to prevent inhibition of chloroplast electron transport activity, is well documented (7). In addition, Reimer et al. (8) have recently shown that BSA/ DBMIB ratios substantially higher than those used by Guikema and Yocum (6) can reverse the high-affinity site of DBMIB inhibition, in an assay system where ferredoxin/NADP was the electron acceptor. We have continued our studies on the interaction between membrane-associated DBMIB and exogenously added BSA. In this communication we report the results of a series of experiments which demonstrate that BSA can remove DBMIB from the high-affinity site on the thylakoid membrane in a reaction which is regulated by the energized state of the membranes. This reaction is unique to BSA; neither other proteins nor thiols we have examined can produce a reversal of 


\section{DBMIB inhibition similar to that reported} here.

\section{MATERIALS AND METHODS}

Spinach chloroplasts were prepared as described by Robinson and Yocum (9) and stored at $-70^{\circ} \mathrm{C}$ in $0.5-$ $\mathrm{ml}$ aliquots prior to assay. Dimeric and monomeric BSA were prepared as follows: $100 \mathrm{mg}$ of BSA was dissolved in $2 \mathrm{ml}$ of $20 \mathrm{~mm}$ Tricine ( $\mathrm{pH}$ 8) containing $20 \mathrm{mM} \mathrm{NaCl}$. The solution was applied to a Sephadex G-200 column $(2.3 \times 65 \mathrm{~cm})$ equilibrated in the buffer used to dissolve the protein. All operations were carried out at $4^{\circ} \mathrm{C}$. The flow rate of the column was adjusted to $20 \mathrm{ml} / \mathrm{h}$, and $1.0-\mathrm{ml}$ fractions were collected and analyzed for absorption at $280 \mathrm{~nm}$. Protein-containing material comprised of oligomeric, dimeric, and monomeric forms of BSA were collected and pooled according to the elution profile. The protein concentration was determined based on an $\xi_{\mathrm{mM}}^{280} \mathrm{~nm}$ of 43.6. Charcoal-defatted BSA was prepared by the procedure of Chen (10), and sulfhydryl-blocked BSA (BSA-CAM) was prepared by the procedure of Nikkel and Foster (11).

Electron transport activity of isolated chloroplasts was assayed by measuring either oygen evolution or uptake with a Clark-type electrode fitted to a thermostatted $\left(25^{\circ} \mathrm{C}\right) 1.5-\mathrm{ml}$ cuvette. The reaction mixture contained $53 \mathrm{~mm}$ Tricine ( $\mathrm{pH} 8), 66 \mathrm{mM} \mathrm{NaCl}, 3.3 \mathrm{mM}$ $\mathrm{MgCl}_{2}, 1.3 \mathrm{mM} \mathrm{KCN}, 66 \mu \mathrm{M}$ methylviologen, and $31 \mu \mathrm{g}$ chlorophyll unless otherwise noted in the legends to the figures. When assays were conducted under conditions to produce unstacking of the thylakoid membranes, both $\mathrm{NaCl}$ and $\mathrm{MgCl}_{2}$ were omitted from the reaction mixture and the chloroplasts were incubated for $2 \mathrm{~min}$ in this low-salt mixture, which has been shown to produce unstacking $(12,13)$ prior to illumination. Membranes were restacked by incubation in the reaction mixture $\left(-\mathrm{NaCl},-\mathrm{MgCl}_{2}\right.$ ) for $20 \mathrm{~min}$ to produce unstacking, after which both $\mathrm{NaCl}(66 \mathrm{mM})$ and $\mathrm{MgCl}_{2}(3.3 \mathrm{mM})$ were added, followed by a 2 -min ineubation period before illumination. Saturating illumination $\left(1 \times 10^{6} \mathrm{ergs} /\right.$ $\mathrm{cm}^{2} \cdot \mathrm{s}$ ) was provided by an Oriel Model 6325 light source filtered through $5 \mathrm{~cm}$ of an $0.2 \%$ solution of $\mathrm{CuSO}_{4}$ and a red cut-on filter $(\lambda>600 \mathrm{~nm})$. Light intensities were varied in some experiments by placing wire mesh screens in the light path, and the resulting intensities were measured at the cuvette surface with a YSI Model 65 Radiometer. Measurements of the light-induced absorbance changes at $518 \mathrm{~nm}$ were made with an Aminco DW-2 dual wavelength spectrophotometer, using the wavelength pair $518-540 \mathrm{~nm}$.

Crystallized, lyophilized BSA (catalog No. A4378) was obtained from Sigma. The following were also obtained from Sigma: Valinomycin, cysteine, lysozyme, jack bean urease, iodoacetamide, and Tricine. Gramicidin was from Mann Research, and DBMIB was a gift from Dr. J. N. Siedow. All other chemicals used in these studies were of the purest grades commercially available.

\section{RESULTS}

At the outset of the experiments reported here, we were interested in determining the cause for our inability to observe reversal by $B S A$ of DBMIB inhibition of photosynthetic electron transport at the high-affinity site, a reversal which Reimer et al. (8) had demonstrated. Whereas these investigators had employed ferredoxin/NADP as an electron acceptor, we had used ferricyanide. It was therefore possible that ferricyanide was in some way interfering with the binding of DBMIB to BSA. That this is in fact true is shown by the data presented in Fig. 1, showing the ability of low concentrations of DBMIB to inhibit electron transport. 'The chloroplast suspensions used for these assays contain $2 \mathrm{mg} / \mathrm{ml} \mathrm{BSA}$; thus, BSA is carried over into the reaction mixture to a final concentration of about $0.2 \mu \mathrm{M}$. Note that when methylviologen is the electron acceptor, the inhibitory potency of DBMIB is decreased in the region from 0 to $0.2 \mu \mathrm{M}$. No such effect was seen when ferricyanide served as the electron acceptor. This result suggested to us that ferricyanide, an anion, might interfere with the interaction between $\mathrm{BSA}$ and DBMIB. As shown in Fig. 1, when ferrocyanide was included in the methylviologen assay system, a treatment which had no effect on the rate of methylviologen reduction, the inhibition of activity by DBMIB was observed to proceed in a fashion identical to that observed in the ferricyanide system. Thus, it appears that (i) DBMIB binding to BSA occurs in the methylviologen system and (ii) either ferricyanide or ferrocyanide can prevent binding. When BSA interference is removed by addition of ferrocyanide, DBMIB is an equally potent inhibitor of both ferricyanide and methylviologen reduction. We calculate from results such as those shown in Fig. 1 that DBMIB has an inhibitory $I_{50}$ value of $0.03 \mu \mathrm{M}$, which indicates that fewer than two molecules of inhibitor are required per electron transport chain to produce a $50 \%$ inhibition of activity, in agreement with other results (14).

In the course of the experiments shown in Fig. 1, we also noted that BSA, when added 


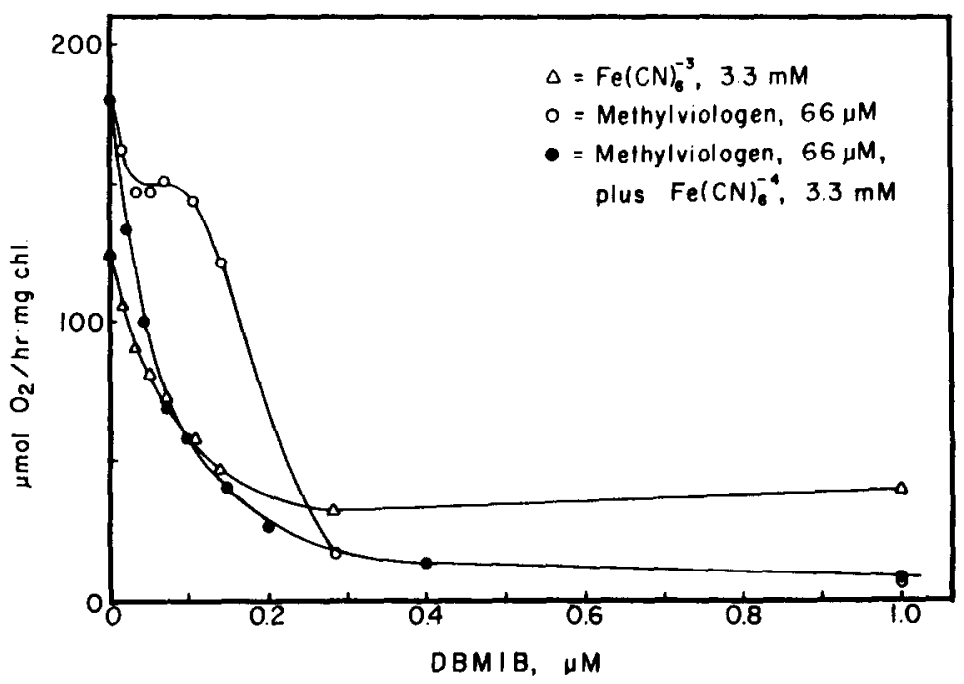

FIG. 1. Inhibition of chloroplast electron transport by DBMIB. Assay conditions are described under Materials and Methods, except that methylviologen was omitted when ferricyanide was used as the electron acceptor, and ADP (1 mM) and inorganic phosphate $(5 \mathrm{~mm})$ were present in all assays. The chloroplasts plus DBMIB were incubated for 1 min prior to illumination.

to the methylviologen assay system after addition of $1 \mu \mathrm{M}$ DBMIB to the chloroplasts, was capable of reversing inhibition of electron transport activity. In contrast to the results of Reimer et al. (8), we observed that the reversal phenomenon was time dependent, rather than immediate. Oxygen electrode tracings from our experiments are shown in Fig. 2, where chloroplasts inhibited by $1 \mu \mathrm{M}$ DBMIB, either in the absence of ADP and inorganic phosphate, or in the presence of gramicidin, were exposed to several concentrations of
BSA at the onset of illumination of the reaction mixture. Several points are illustrated by these results. First, addition of BSA does not, under these conditions of assay, produce an immediate reversal of inhibition. Rather, there is a period where no electron transport activity is observed, followed thereafter by the appearance of a low rate of oxygen uptake which is then superceded by uninhibited activity. Second, the times for reversal to occur (as determined by the extrapolation, shown in Fig. 2, from the points of intersection of the slopes of the

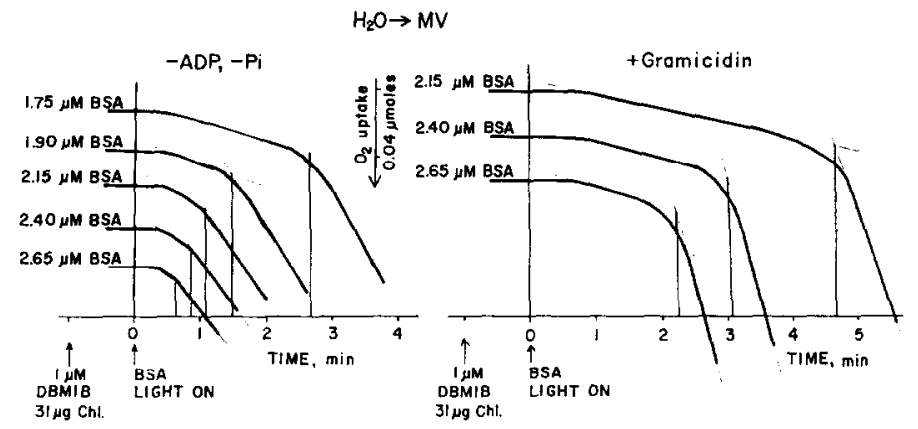

FIG. 2. Time dependence of the restoration of electron transport in DBMIB-inhibited chloroplasts with varying concentrations of BSA. Assay conditions are given under Materials and Methods. Chloroplasts plus $1 \mu \mathrm{M}$ DBMIB were incubated for $1 \mathrm{~min}$ in the dark in the reaction mixture prior to the simultaneous injection of RSA and the onset of illumination. Gramicidin was present where indicated at a concentration of $5 \mu \mathrm{g} / 1.5 \mathrm{ml}$ of assay medium. 


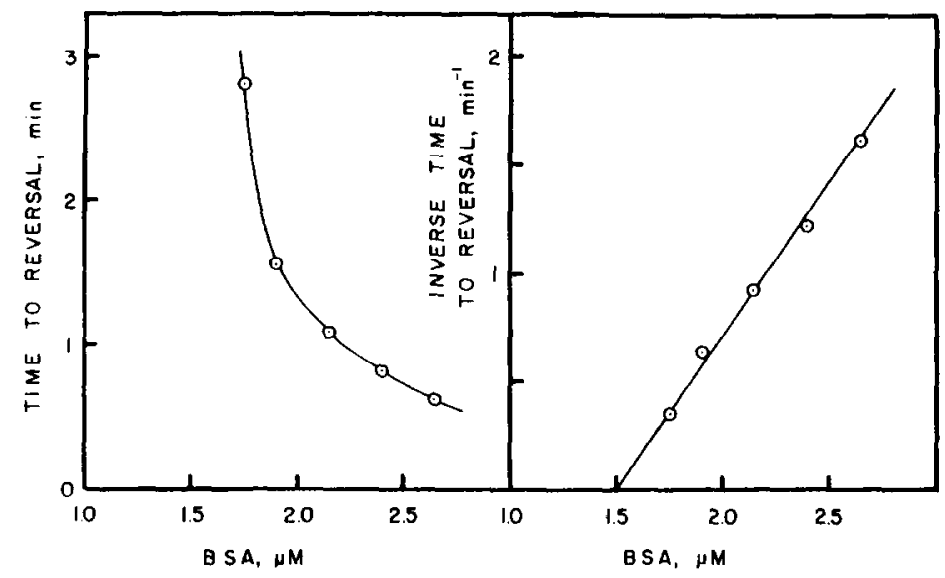

FIG. 3. Plots of time to reversal of inhibition and inverse time to reversal as a function of BSA concentration. The data are derived from the experiment shown in Fig. 2, conducted in the absence of ADP and inorganic phosphate. See text for further details.

low and high rates of electron transport activity), are seen to be dependent on the concentration of $\mathrm{BSA}$ added to the reaction mixture. Last, the time required in the light for reversal to occur is governed by the extent of energization of the membranes, as evidenced by the ability of gramicidininduced uncoupling to prolong the duration of the inhibited state.

Two methods for plotting the data in Fig. 2 are shown in Fig. 3 . When the time required for full reversal is plotted as a func-

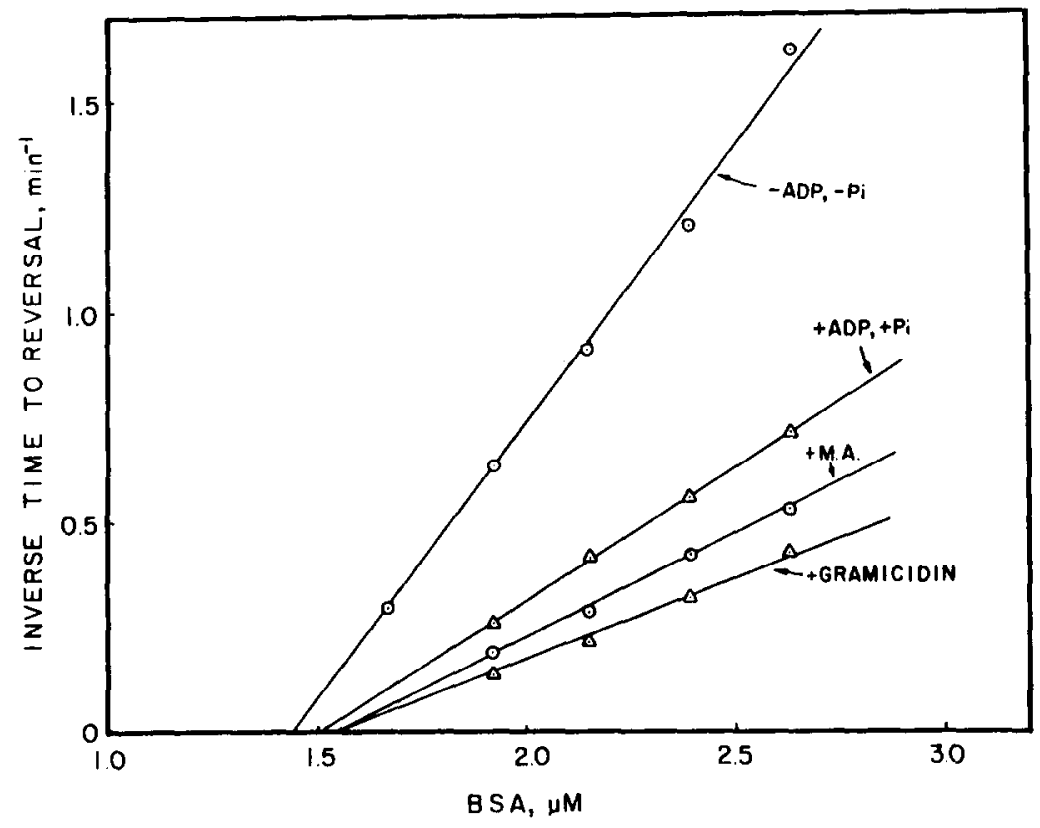

FIG. 4. The effect of membrane energization on the time required for reversal of DBMIB inhibition of electron transport by BSA. Assay conditions are described in the legend to Fig. 2. Additions to the assays were, where indicated, $1 \mathrm{~mm}$ ADP, $5 \mathrm{~mm}$ inorganic phosphate, $5 \mu \mathrm{g}$ gramicidin, and $10 \mathrm{~mm}$ methylamine; ADP and inorganic phosphate were also present in the experiments where uncouplers were used. 


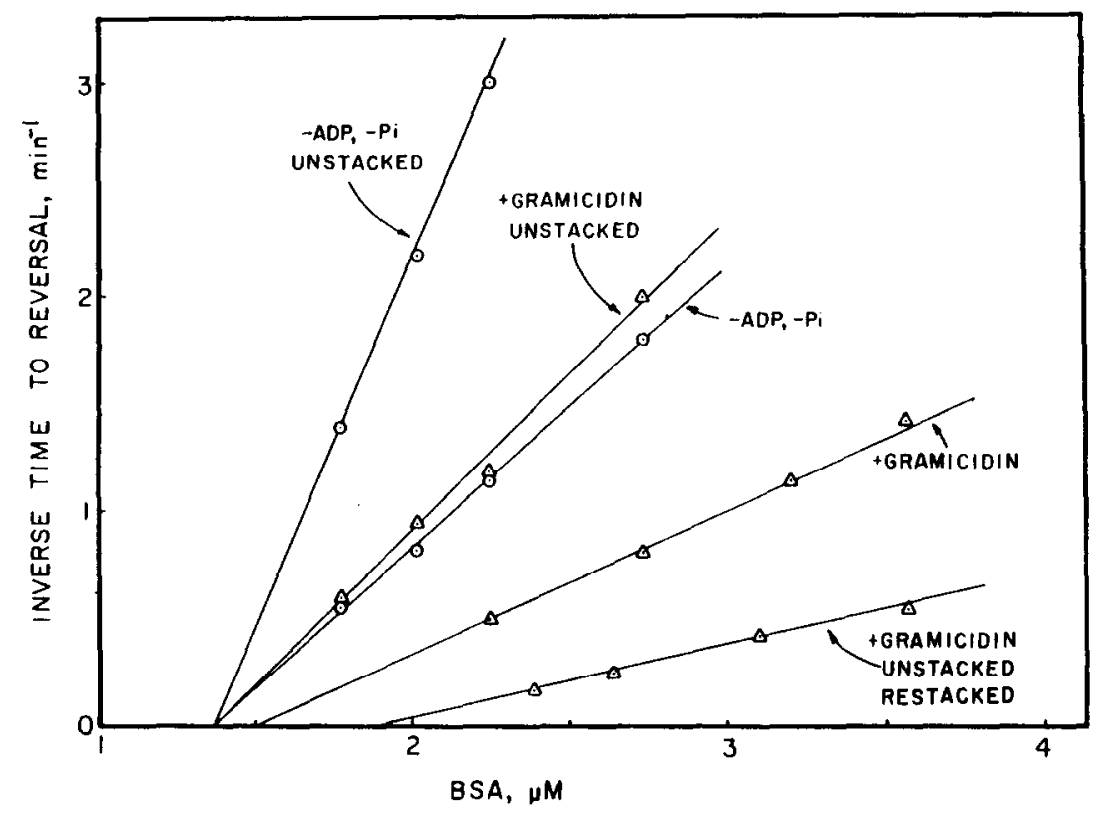

Fig. 5. Effect of exposure of chloroplast membranes to low salt conditions on the reversal of DBMIB inhibition. Assay conditions are given in the legend to Fig. 2 and under Materials and Methods. In addition, ADP and inorganic phosphate were omitted from the experiments where gramicidin was present.

tion of the concentration of BSA added to the assay system, the result shown in Fig. 3 (left) is obtained; if the reciprocal of the reversal time is used instead, the linear plot shown in Fig. 3 (right) is produced. Extrapolation of this plot to the abscissa (infinite time) provides an estimate of the minimum concentration of BSA required to reverse $D B M I B$ inhibition, while the slope of this line provides an indication of the rate of reversal of inhibition. This method (Fig. 3 (right)) for plotting the results of inhibition reversal experiments was applied to the analysis of data generated in a number of experiments in which chloroplasts inhibited with $1 \mu \mathrm{M}$ DBMIB were exposed to both varying concentrations of $\mathrm{BSA}$ and varying conditions of assay with regard to the degree of membrane energization. The results of these experiments, shown in Fig. 4 , indicate that although the abscissa intercept is subject to little variation by the conditions of assay, the time required for reversal of DBMIB inhibition of electron transport by added BSA, as monitored by the slopes of the lines in Fig. 4, is regulated by the energized state of the thylakoid mem- branes during illumination. In the absence of ADP and inorganic phosphate, reversal is relatively rapid; longer time requirements are imposed on the reversal process by addition of ADP plus inorganic phosphate, as well as by the addition of uncouplers (gramicidin, methylamine). Thus the energized state of the chloroplast membrane appears to regulate the time required for reversal to occur, but not the amount of BSA minimally required to effect reversal (the abscissa intercept of Fig. 4).

There are two probable explanations for the results shown in Fig. 4. Either the energization of the thylakoid membranes generates a change in the structure of the membrane (perhaps shrinking or swelling), which enhances the ability of BSA to interact with membrane-associated DBMIB, or the presence of a membrane potential is acting to promote the inhibitor-protein interaction. The first of these possibilities (a gross structural change in the membrane) was tested with unstacked chloroplasts produced by placing membranes in a low-salt medium (Fig. 5). Results obtained with stacked membranes are included in this 


\section{TABLE I}

EFFECTS OF VALINOMYCIN ON THE 518-nm absorbance Change and Electron TRansport ACtivitY ${ }^{a}$

\begin{tabular}{ccc}
\hline $\begin{array}{c}\text { Valinomycin } \\
(\mu \mathrm{M})\end{array}$ & $\begin{array}{c}\text { Extent of } \\
518 \text { change } \\
(\%)\end{array}$ & $\begin{array}{c}\text { Electron transport rate } \\
\left(\mu \mathrm{mol} \mathrm{O}_{2} / \mathrm{h} \cdot \mathrm{mg} \mathrm{chl}\right)\end{array}$ \\
\hline 0 & 100 & 97 \\
0.05 & 49 & 80 \\
0.10 & 28 & 77 \\
0.25 & 15 & 70
\end{tabular}

\footnotetext{
${ }^{a}$ The 518-nm absorbance change and electron transport activities were measured as described under Materials and Methods, with the addition of $10 \mathrm{mM}$ KCl. The uninhibited extent $(100 \%)$ of the 518-nm change was $0.008 \mathrm{~A}$. Absorbance changes were recorded on a single averager, and the amplitude was determined from the change observed upon the opening of the electronic shutter to avoid slower changes due to scatter. The time constant for the system was $5 \mathrm{~ms}$ for $63 \%$ of full-scale response.
}

figure for purposes of comparison. An increased rate of reversal of inhibition is observed with unstacked membranes, even in the presence of gramicidin, although unstacked, uncoupled chloroplasts show a longer time requirement for reversal to occur. These reversal times are more rapid than those observed with stacked chloroplast membranes assayed under the same conditions, and restacking of the membranes, also shown in Fig. 5, generates a requirement for a longer illumination time to produce reversal.

The possible role of a membrane potential in regulating BSA-DBMIB interactions was also examined using valinomycin plus KCl. Since Voegeli et al. (15) had shown that valinomycin was an inhibitor of electron transport between photosystem II and photosystem I at a site similar to that affected by DBMIB, we compared the effects of the ionophore both on the 518-nm carotenoid bandshift, which monitors membrane potential (16), and on the rate of photosynthetic electron transport in our chloroplast preparations. The data from these experiments (Table I) show that valinomycin at the concentrations used here cannot abolish the 518-nm absorption change with- out concomitantly blocking some electron transport activity. With this finding in mind, we examined the effect of valinomycin plus $\mathrm{KCl}$ on the ability of BSA to reverse DBMIB inhibition (Table II). The results shown here indicate that valinomycin can prolong the time neccssary for BSA to reverse DBMIB inhibition. However, this effect is not necessarily due entirely to an effect on the membrane potential; reversal of inhibition in gramicidin-uncoupled chloroplast membranes is also retarded by valinomycin (Table II).

The effect of incident light intensity on the assay system during reversal of DBMIB inhibition of electron transport by BSA was also examined, in an effort to determine if limiting electron transport activity (at low light intensities) would produce an effect on the time required for reversal to occur. A summary of these experiments, presented in Table III, shows that the intensity of illumination during the reversal process does not influence the times required for appearance of the uninhibited rate of electron transport, even though these rates are attenuated at the lower intensities. Similar results were obtained with gramicidin-uncoupled chloroplasts (data not shown).

\section{TABLE II}

EFFECTS OF VALINOMYCIN ON THE TIME REQUIREMENT FOR BSA TO REVERSE DBMIB INHIBITION $^{a}$

\begin{tabular}{lcc}
\hline & $\begin{array}{c}\text { Time to } \\
\text { reversal } \\
\text { (min) }\end{array}$ & $\begin{array}{c}\text { Rate of electron } \\
\text { transport after } \\
\text { reversal }(\mu \mathrm{mol} \\
\mathrm{O}_{2} / \mathrm{h} \cdot \mathrm{mg} \text { chl) }\end{array}$ \\
\hline None & 1.7 & 112 \\
$\mathrm{KCl}$ & 1.9 & 115 \\
Val $(0.05 \mu \mathrm{M})+\mathrm{KCl}$ & 3.6 & 129 \\
Val $(0.10 \mu \mathrm{M})+\mathrm{KCl}$ & 5.3 & 110 \\
Val $(0.10 \mu \mathrm{M})$ & 3.5 & 128 \\
Gram $+\mathrm{KCl}$ & 3.5 & 180 \\
Gram $+\mathrm{Val}(0.10 \mu \mathrm{M})$ & & 128 \\
$\quad \mathrm{KCl}$ & 5.3 & \\
\hline
\end{tabular}

a Assay conditions are as described in the legend for Fig. 2, except that $\mathrm{NaCN}$ replaced KCN. The BSA concentration was $3.2 \mu \mathrm{M}$; when present, the concentrations of $\mathrm{KCl}$ and gramicidin were $10 \mathrm{mM}$ and $5 \mu \mathrm{g} / 1.5 \mathrm{ml}$, respectively. All assays were conducted in the absence of ADP and inorganic phosphate. 
TABLE III

EFFECT OF ILLUMINATION INTENSITY ON THE BSA REVERSAL OF DBMIB INHIBITION OF ELECTRON

TRANSPORT ACTIVITY ${ }^{a}$

\begin{tabular}{ccc}
\hline $\begin{array}{c}\text { Intensity } \\
\left(\mathrm{kerg} / \mathrm{cm}^{2} \cdot \mathrm{s}\right)\end{array}$ & $\begin{array}{c}\text { Time to } \\
\text { reversal } \\
(\mathrm{min})\end{array}$ & $\begin{array}{c}\text { Rate of electron transport } \\
\left(\mu \mathrm{mol} \mathrm{O}_{2} / \mathrm{h} \cdot \mathrm{mg} \mathrm{chl}\right)\end{array}$ \\
\hline 1070 & 1.9 & 124 \\
140 & 1.7 & 89 \\
54 & 1.7 & 60 \\
\hline
\end{tabular}

${ }^{a}$ Conditions and procedures used for assay are described in the legend to Fig. 2 (assayed in the absence of ADP and inorganic phosphate). The BSA concentration was $2.5 \mu \mathrm{M}$.

The results presented thus far have sought to characterize the process by which BSA reverses DBMIB inhibition of electron transport in terms of changes in the thylakoid membranes, and the electron transport activity associated with them. We have also sought to characterize this reversal phenomenon in terms of modifications of BSA. Commercially available BSA, such as that used in these studies, contains some dimerized protein which can be isolated by gel filtration, as described under Materials and Methods. Figure 6 compares the abilities of both purified monomeric and dimeric species of BSA to reverse DBMIB inhibition of electron transport activity. Although the dimer is able to effect reversal, the reversal process requires longer times in the light, and higher concentrations of dimer are necessary, as evidenced by the change in the abscissa intercept shown in Fig. 5. Figure 7 presents the results of reversal assays carried out with charcoal-defatted BSA (10), and with BSA treated with iodoacetamide to block the free - SH group on the protein (11). Neither defatting nor carboxymethylation has an appreciable effect on the ability of BSA to reverse electron transport inhibition. In the case of the protein treated with iodoacetamide, the free - SH group is shown not to be required for reversal to occur (17).

We have also compared the efficacy, in reversal of DBMIB inhibition, of a number of compounds, shown in Table IV; BSA is included here for purposes of comparison. These data show that both thiols (DTT, cysteine) and proteins (lysozyme, jack bean urease) are capable of releasing the inhibi-

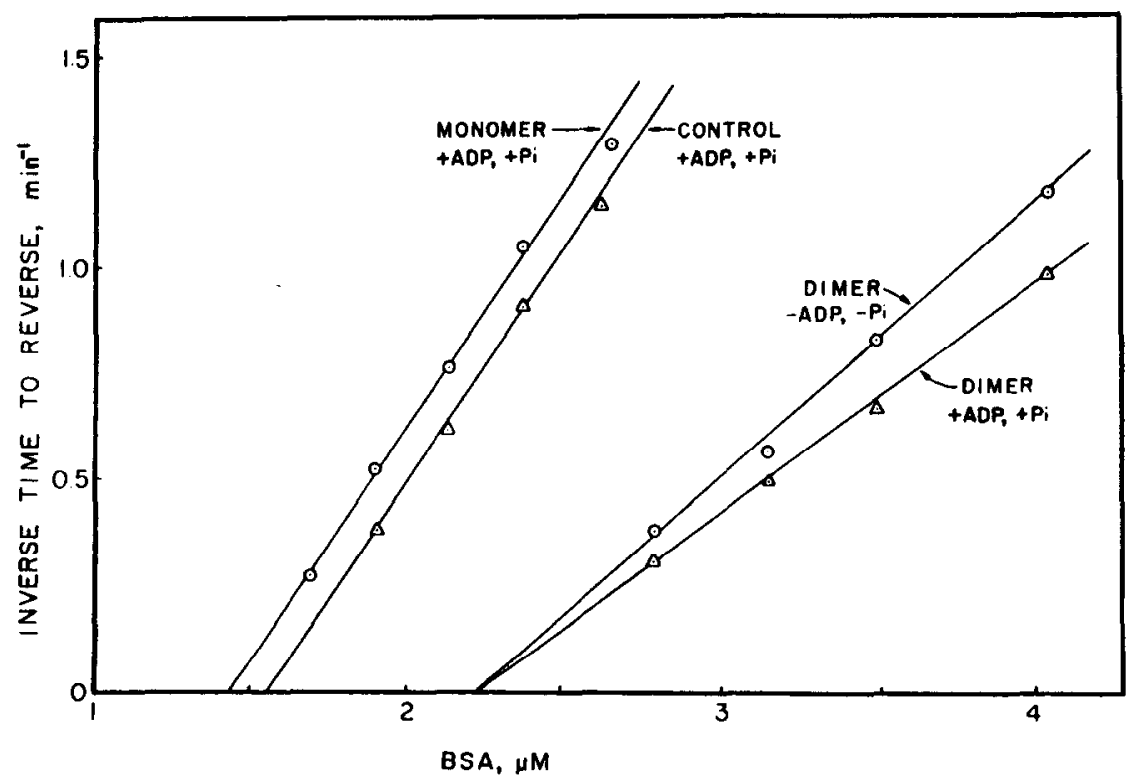

FIG. 6. Comparison of the efficacies of monomeric and dimeric BSA in reversing DBMIB inhibition. Conditions of assay are as given in the legend to Fig. 2, with ADP (1 mM) and inorganic phosphate (5 mM) present. 


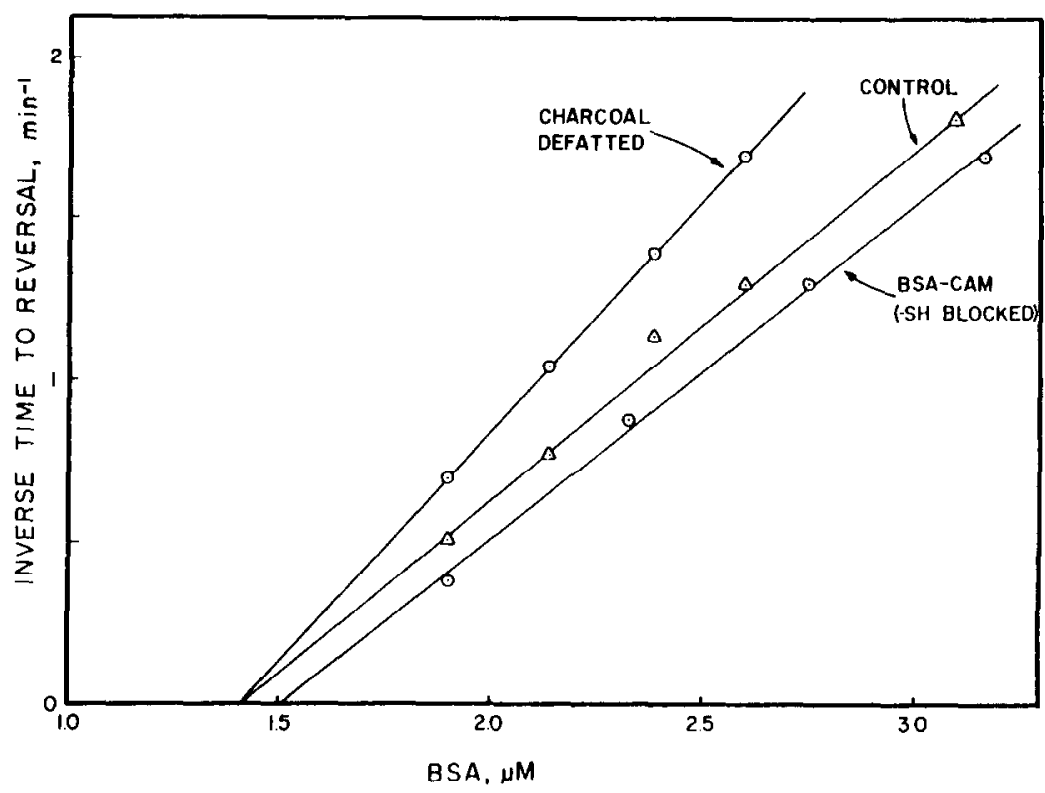

FIG. 7. Effects of defatting and carboxymethylation on the ability of BSA to reverse DBMIB inhibition. Assay conditions are given in the legend to Fig. 6 .

tion imposed on electron transport by DBMIB, although with altered kinetics (data not shown). Note, however, that reversal of inhibition by these compounds is invariably slower than with BSA, and also that the response to the energized state of the thylakoid membranes is most pronounced when BSA is the agent used to reverse inhibition. These results suggest the existence of a unique interaction between BSA and DBMIB; other data presented earlier (the abscissa intercepts of Figs. 3-7) also indicate that some stoichio- metric relationship exists between BSA and DBMIB which is reflected in the times required for optimal recovery of the electron transport apparatus from inhibition. That such an interaction exists is confirmed by the data shown in Fig. 8. These experiments involved the assay of inhibition reversal using the concentrations of DBMIB shown in the figure. Unstacked chloroplasts were used to minimize both the time required for reversal and thus the possible damage to photoactivity caused by the prolonged illumination times required at high DBMIB

TABLE IV

Reversal of DBMiB Inhibition By Thiols and Proteins ${ }^{a}$

\begin{tabular}{|c|c|c|c|c|c|}
\hline \multirow[b]{2}{*}{ Condition of assay } & \multicolumn{5}{|c|}{ Time ( $\mathrm{min}$ ) required for inhibition reversal in the presence of } \\
\hline & Cysteine & DTT & Lysozyme & Urease & BSA \\
\hline$-\mathrm{ADP},-\mathrm{P}_{1}$ & 11.8 & $>15$ & 4.1 & 4.7 & 0.25 \\
\hline$+5 \mu \mathrm{g}$ Gram & 11.0 & $>15$ & 3.2 & 5.3 & 0.50 \\
\hline Unstacked, - ADP, $-P_{\mathbf{i}}$ & 6.8 & $>15$ & 1.7 & 4.1 & 0.20 \\
\hline Unstacked, $+5 \mu \mathrm{g}$ Gram & 5.8 & $>15$ & 1.6 & 5.0 & 0.35 \\
\hline
\end{tabular}

a Assay conditions are given in the legend for Fig. 2 and under Materials and Methods. Thiols or proteins were injected to give a final concentration in the reaction mixture of $10 \mu \mathrm{M}$. The response time of the oxygen electrode used in these assays was $0.15 \mathrm{~min}$. The reactions where DTT was present were terminated after 15 min of illumination. 
concentrations. The results of these experiments establish the existence of a stoichiometric relationship (approximately $1.5 \mu \mathrm{mol}$ $\mathrm{BSA} / \mu \mathrm{mol} \mathrm{DBMIB}$ ) in the reversal process.

\section{DISCUSSION}

The data presented under results document the ability of BSA to reverse the inhibition by DBMIB of photosynthetic electron transport. This reversal process is complex, and is comprised of the restoration first of a low rate of electron flow followed thereafter by the appearance of the uninhibited rate of methylviologen reduction (Fig. 2). The failure of Reimer et al. (8) to observe this phenomenon may be due to the fact that these investigators used high concentrations of BSA added to osmotically shocked chloroplasts. A further complexity associated with the reversal process is revealed by our observation that the time required for reversal to occur is governed by the energized state of the chloroplast membranes in the light (Figs. 2 and 4). Conditions under which the thylakoids should be maximally energized (illumination in the absence of ADP and inorganic phosphate) are those conditions which generate the shortest time requirements for reversal to occur; these times are significantly lengthened by the inclusion of either ADP and inorganic phosphate, or of uncouplers, in the assay medium. Furthermore, the reversal phenomenon appears to be unique to the BSADBMIB interaction, as evidenced by the failure of either other proteins or thiols to produce a similar effect (Table IV).

Energization by illumination of thylakoid membranes has been shown by a number of investigators $(13,18-20)$ to induce structural changes in these membranes (shrinking, swelling). We have sought to correlate such changes with the reversal phenomenon reported here. Unstacking of the thylakoid membranes, a swelling effect, dramatically decreases the time required for BSA to reverse DBMIB inhibition (Fig. 5), suggesting in turn that some light-induced swelling of membranes during energization could serve to regulate the ability of BSA to interact with DBMIB. In this regard, the findings with methylamine (Fig.

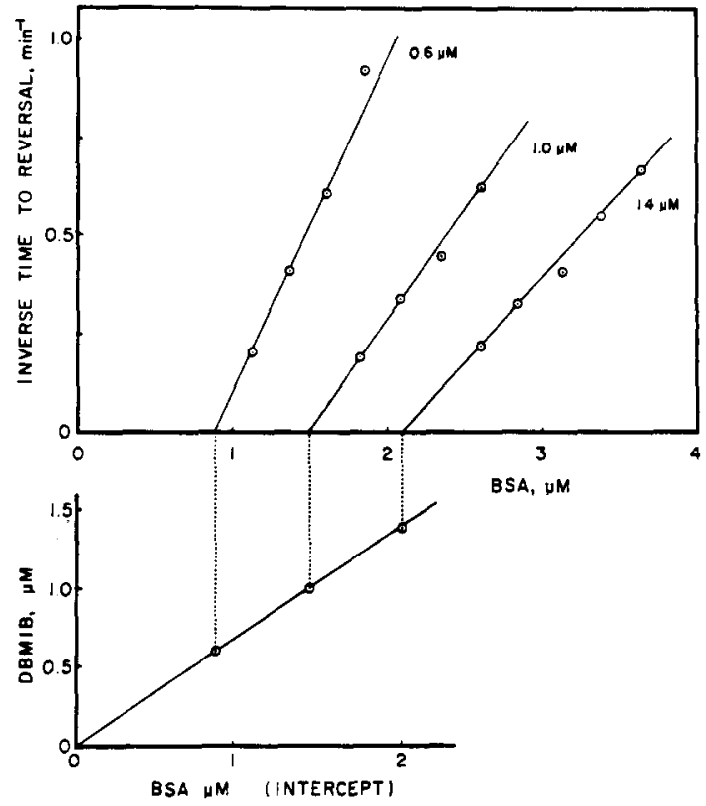

FIG. 8. Linearity of the abscissa intercept with varying concentrations of DBMIB. Unstacked chloroplasts were used for assay, and $5 \mu \mathrm{g}$ of gramicidin was present in all assays. Other conditions of assay are given in the legend to Fig. 2.

4), which should induce thylakoid swelling by amine uptake (18), cannot be reconciled to a model in which expansion of membrane volume by itself promotes access of BSA to membrane-associated DBMIB. The ability of both methylamine and gramicidin to prolong the illumination times required for reversal of DBMIB inhibition to occur suggests that $\Delta \mathrm{pH}$ and/or a membrane potential might play a role in facilitating reversal. Attempts to assess a possible contribution of membrane potential to the reversal process have produced ambiguous results, owing to the ability of valinomycin to inhibit electron transport activity (Table I). It is certainly possible that the change in surface potential which occurs upon illumination of thylakoids (21) could in turn affect the ability of BSA to react with membrane-associated DBMIB, and our data do not eliminate this possibility.

Our results indicate that DBMIB is removed from its site of inhibition on or in the thylakoid membrane by means of a specific interaction between the inhibitor and BSA. 
We have demonstrated the existence of a stoichiometry between BSA and DBMIB in the reversal process (Fig. 8), and the ability of BSA to bind DBMIB in solution has been documented $(7,8)$. It is therefore reasonable to assume that reversal of inhibition is occurring by the removal of DBMIB from the membrane by binding to BSA, rather than by some chemical modification of the inhibitor on the membrane. Furthermore, it seems likely that BSA must make contact with the membrane to effect reversal. These proposals are supported by our observations that carboxymethylation of BSA to block the - SH group does not interfere with the reversal reaction (Fig. 7); debromination by the $-\mathrm{SH}$ group (17) is thus eliminated as a cause of the reversal process. The finding that a larger protein species, namely dimeric $\mathrm{BSA}$, can also reverse inhibition, but at a much slower rate than can the smaller monomeric species (Fig. 6), suggests that the monomer can approach the membrane with greater facility than can the dimer.

These considerations raise the question of the topological location of the DBMIB inhibition site on, or in, the thylakoid membrane, and of the nature of the mechanisms which act to regulate access of BSA to this site. It is clear from our data that some portion of the electron transport chains inhibited by DBMIB must be readily accessible to BSA in order to explain the appearance, after a short period of illumination, of low rates of electron transport activity. A greater proportion of the inhibition sites must exist in locations shielded from the external medium, however, and access to BSA to these sites is promoted by energylinked changes which occur upon illumination of the membrane. These changes may relate to the position of the inhibitory site on the membrane, perhaps in the appressed regions of grana stacks, where energization produces structural changes, as yet undefined, to permit contact between BSA and the site of DBMIB inhibition. Alternatively, energization could produce alterations in the surface charge of the membrane which facilitate the approach of BSA to the in- hibitory site. Either explanation seems likely at present, and it is equally probable that both phenomena are acting in concert to produce reversal of inhibition. Further studies employing anions and cations are currently in progress in an attempt to resolve these questions.

\section{REFERENCES}

1. Trebst, A., Harth, E., and Draber, W. (1970) Z. Naturforsch. 25b, 1157-1159.

2. BOHME, H., Reimer, S., AND TreBst, A. (1971) Z. Naturforsch. 26b, 341-352.

3. DE Kouchkovsky, Y. (1974) in Proceedings of the Third International Congress on Photosynethesis (Avron, M., ed.), pp. 1081-1093, Elsevier, Amsterdam.

4. Trebst, A., AND ReImer, S. (1973) Z. Naturforsch. 28c, 710-716.

5. Stewart, A., and Larkum, A. W. D. (1977) Aust. J. Plant Physiol. 4, 253-261.

6. Guikema, J. A., ANd Yocum, C. F. (1978) Arch. Biochem. Biophys. 189, 508-515.

7. Berg, S. P., AND IzaWA, S. (1977) Biochim. Biophys. Acta 460, 206-219.

8. Reimer, S., Link, K., AND Trebst, A. (1979) Z. Naturforsch. 34c, 419-426.

9. Robinson, H. H., ANd Yocum, C. F. (1980) Biochim. Biophys. Acta. 590, 97-106.

10. Chen, R. F. (1967) J. Biol. Chem. 242, 173-181.

11. NikKel, H. J., AND Fos'TER, J. F. (1971) Biochemistry 10, 4479-4486.

12. Murakami, S., and Packer, L. (1971) Arch. Biochem. Biophys. 146, 337-347.

13. Izawa, S., AND GoOd, N. E. (1966) Plant Physiol. 41, 533-543.

14. HaEhNeL, W. (1977) in Bioenergetics of Membranes (Packer, L., et al., ed.), pp. 317-328, Elsevier, Amsterdam.

15. Voegel, K. K., O'Keefe, D., Whitmarsh, J., AND DILley, R. A. (1977) Arch. Biochem. Biophys. 183, 333-339.

16. WITT, H. T. (1979) Biochim. Biophys. Acta 505, $355-427$.

17. Reimer, S., AND Trebst, A. (1976) Z. Naturforsch. 31c, 103.

18. Deamer, D. W., Crofts, A. R., AND Packer, L. (1967) Biochim. Biophys. Acta 131, 81-96.

19. Crofts, A. R., Deamer, D. W., and Packer, L. (1967) Biochim. Biophys. Acta 131, 97-118.

20. NoBel, P. S. (1967) Biochim. Biophys. Acta 131, 127-140.

21. QUINTANILhA, A. T., AND PACKeR, L. (1978) Arch. Biochem. Biophys. 190, 206-209. 